\title{
COHOMOLOGY RINGS OF A CLASS OF TORUS MANIFOLDS
}

\author{
SOUMEN SARKAR AND DONALD STANLEY
}

\begin{abstract}
Torus manifolds are topological generalization of smooth projective toric manifolds. We compute the rational cohomology ring of a class of smooth locally standard torus manifolds whose orbit space is a connected sum of simple polytopes.
\end{abstract}

\section{INTRODUCTION}

Topological generalization of non-singular smooth projective toric varieties first appeared in the pioneer work of Davis and Januszkiewicz [DJ91] with the name 'toric manifold'. These spaces are also known as quasitoric manifolds [BP02, Chapter 5]. Further generalizations like torus manifolds, manifolds with local torus action and topological torus manifolds have appeared in [HM03], [Yos11] and [IFM13]. The study of torus actions on manifolds and orbifolds sparks many interesting developments. Briefly, A torus manifold is an even dimentional manifold with an effective half-dimensional torus action having non-empty fixed points. In addition, if the local representation resembles the standard action, then it is known as a locally standard torus manifold. An example of a locally standard torus manifold which is not toric manifold is even dimensional sphere $S^{2 n}$ with the standard action of $T^{n}$, see Example 2.10. Many topological invariants of locally standard torus manifolds are known, for example [MP06, AMPZ17], [Ayz16a, Ayz16b], but mainly they consider the corresponding orbit space is face acyclic or the proper faces of the orbit space are acyclic. The face acyclic condition on the orbit space leads to trivial cohomology in odd-degrees of the torus manifold. [MP06, Theorem 4.1] says that if the odd-degree cohomology of a torus manifold is trivial then the torus action is locally standard.

In this article we investigate the cohomological properties of torus manifolds where the associated orbit space may have non-acyclic proper faces. We consider torus manifolds which may be obtained by equivariant gluing of toric manifolds along deleted neighborhood of nontrivial torus orbits, see Section 2. We remark that more general connected sums may be found in [GK98]. In this section, we show that if the orbit space of a locally standard torus manifold $M$ is a connected sum of simple polytopes then $M$ is an equivariant connected sum of toric manifolds, see Lemma 2.12. Moreover, in Lemma 2.13 we show that an equivariant connected sum of two toric manifolds is homemorphic to a connected sum of two toric manifolds and one locally standard torus manifold. In Section 3, we study the homotopy type of the

2010 Mathematics Subject Classification. 55N99, 57R99, 52B05.

Key words and phrases. polytopes, torus action, torus manifold, (equivariant) connected sum, homology groups, cohomology ring. 
complement of an orbit of the natural $T^{n}$-action on $S^{2 n}$. We show here that an orbit complement of $S^{2 n}$ is homotopic to a wedge of some lower dimensional spheres. Our equivariant gluing is not at a fixed point. That means the torus manifolds considered here have nontrivial homology in odd-degrees, see Section 4 . In this section we compute the integral homology of equvariant connected sum $S^{2 n} \#_{T^{k}} S^{2 n}$ of two $S^{2 n}$ along a non-trivial orbit $T^{k} \hookrightarrow S^{2 n}$. Section 5 contains the following main theorem where $\mathbb{K}$ is a field of characteristic zero.

Theorem 1.1. Let

$$
A=s^{-1} \widetilde{H}^{*} T^{k} \oplus s^{-2 n+1}\left(\widetilde{H}^{*} T^{k}\right)^{\#} \oplus \mathbb{K} 1 \oplus \mathbb{K} \mu_{2 n}
$$

be a commutative differential graded algebra with the differential $d=0$ and a multiplication $\beta: A \otimes A \rightarrow A$ which is determined by

$$
\begin{aligned}
& \beta\left(s^{-1} \alpha_{I}, s^{-1} \alpha_{J}\right)=0, \beta\left(s^{-1} \alpha_{I}, s^{-2 n+1} \alpha_{J}^{\#}\right)=-\delta_{I J} \mu_{2 n}, \beta(1, x)=x, \\
& \beta\left(s^{-2 n+1} \alpha_{I}^{\#}, s^{-2 n+1} \alpha_{J}^{\#}\right)=0, \text { and } \beta\left(\mu_{2 n}, y\right)=0 \text { for } y \neq 1, \text { with } I, J \subseteq\{1, \ldots, k\} .
\end{aligned}
$$

where $\left\{\alpha_{I}\right\}$ is a graded basis for $\widetilde{H}^{*} T^{k}$ for $I \subseteq\{1, \ldots, k\}$. Then $A$ is a model for the torus manifold $S^{2 n} \#_{T^{k}} S^{2 n}$.

The proof of this theorem follows from Propositions 5.9 and 5.12. At the end of this section we give a presentation of the cohomology ring of locally standard torus manifolds over connected sum of simple polytopes, see Theorem 5.13.

\section{BASICS OF TORUS MANIFOLDS}

2.1. Connected sum of manifolds with corners. A polytope is the convex hull of a finite set of points in $\mathbb{R}^{n}$. An $n$-dimensional polytope is said to be simple if every vertex is the intersection of exactly $n$ codimension one faces. Ready examples of simple polytopes are simplices and cubes. Manifold with corners and manifold with faces are discussed in detail in Section 6 of [Dav83]. Here by manifold with corners we will refer nice manifold with corners. A class of examples of manifolds with corners are simple polytopes. More properties on manifold with corners can be found in [Joy12].

Now we discuss connected sum of manifolds with corners at a relative interior point of faces. Let $P$ and $Q$ be two $n$-dimensional oriented manifolds with corners in $\mathbb{R}^{n}$. Let $F$ and $G$ be $k$-dimensional $(k \geq 0)$ faces of $P$ and $Q$ respectively. Let $x_{F}$ and $y_{G}$ belong to the relative interior of $F$ and $G$ respectively. Let $B$ and $C$ be open $n$-ball around $x_{F}$ and $y_{G}$ respectively in $\mathbb{R}^{n}$ such that

(1) $B \cap P$ and $C \cap Q$ are homeomorphic as manifold with corners to $\mathbb{R}^{k} \times \mathbb{R}_{\geq 0}^{n-k}$.

(2) $\bar{B} \cap H$ is empty for any face $H$ of $P$ not containing $F$.

(3) $\bar{C} \cap K$ is empty for any face $K$ of $Q$ not containing $G$.

Then $\partial \bar{B} \cap P$ and $\partial \bar{C} \cap Q$ are homeomorphic as manifold with corners. Identifying $\partial \bar{B} \cap P$ and $\partial \bar{C} \cap Q$ via an oriented reversing homeomorphism, we get a new oriented manifold with corners, denoted by $P \#_{x_{F}, y_{G}} Q$. 
If $P, Q$ are simple polytopes and $F, G$ are vertices of $P, Q$ respectively, then the connected sum $P \#_{x_{F}, y_{G}} Q$ is homeomorphic as manifold with corners to a simple polytope, see Construction 1.13 in [BP02].

If $P, Q$ are simple polytopes and $F=P$ and $G=Q$, then the connected sum $P \#_{x_{F}, y_{G}} Q$ is diffeomorphic as manifold with corners to a polytope with simple holes, see Subsection 2.1 in [PS15].

Example 2.1. Some polytope with simple holes in $\mathbb{R}^{2}$ are given in Figure 1 . The first figure is the connected sum of a rectangle and a triangle at their interior points. The second figure is the connected sum of an octagon, a rectangle and a triangle at their interior points.
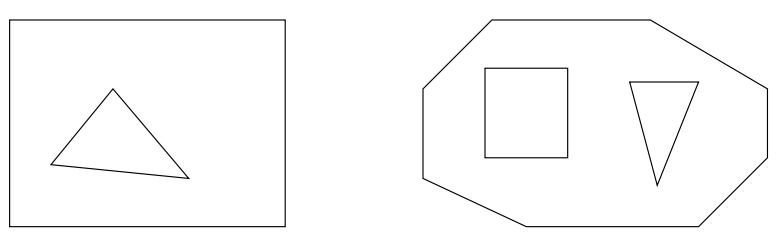

Figure 1. Polytopes with simple holes in $\mathbb{R}^{2}$.

Remark 2.2. $\partial \bar{B} \cap P$ is contractible if and only if dimension of $F$ is less than $n$. So $P \#_{x_{F}, y_{G}} Q$ is contractible if and only if dimension of $F$ is less than $n$ and $P, Q$ are contractible.

Lemma 2.3. Let $P$ be an $n$-dimensional polytope with simple holes. Then $H^{2}(P, \mathbb{Z})$ is trivial if and only if $n \neq 3$.

Proof. Let $P$ be an $n$-dimensional polytope with $s$ simple holes. So $P$ is homotopic to wedge of $s$ many $(n-1)$-dimensional sphere. Lemma follows from this.

Now we discuss another type of connected sum of manifolds with corners at faces. Let $P$ and $Q$ be two $n$-dimensional oriented manifolds with corners in $\mathbb{R}^{n}$. Let $F$ and $G$ be $k$-dimensional $(k \geq 1)$ contractible faces of $P$ and $Q$ respectively. Let $B$ and $C$ be tubular neighborhood of $F$ and $G$ in $P$ and $Q$ respectively such that

(1) $P-B$ and $Q-C$ are manifold with corners in $\mathbb{R}^{n}$.

(2) $B$ and $C$ are homeomorphic as manifold with corners to the sets $F \times \mathbb{R}_{\geq 0}^{n-k}$ and $G \times \mathbb{R}_{\geq 0}^{n-k}$ respectively.

Then $\bar{B} \cap(P-B)$ and $\bar{C} \cap(Q-C)$ are homeomorphic as manifold with corners. Identifying $\bar{B} \cap(P-C)$ and $\bar{C} \cap(Q-C)$ via a homeomorphism, we get a new oriented manifold with corners, denoted by $P \#_{F, G} Q$. Using Van-Kampen theorem for fundamental group, one can show the following.

Lemma 2.4. If $P$ and $Q$ are contractible manifold with corners, then $P \#_{x_{F}, y_{G}} Q$ is contractible. In addition, if $F, G$ are contractible then $P \#_{F, G} Q$ is contractible manifolds with corners. 
2.2. Combinatorial construction. Let $P$ be an $n$-dimensional oriented manifold with corners in $\mathbb{R}^{n}$. Let $\mathcal{F}(P)=\left\{F_{1}, F_{2}, \ldots, F_{m}\right\}$ be the set of all codimension one faces (facets) of $P$. Note that if $F$ is a nonempty face of $P$ of codimension $k$ then $F$ is a connected component of the intersection of a unique collection of $k$ facets of $P$. The following definition is a straightforward generalization of the notion of characteristic function for a simple polytope, which is a crucial concept for studying toric manifolds [DJ91, BP02].

Definition 2.5. [HM03] A closed, connected, oriented, smooth manifold $Y$ of dimension $2 n$ with an effective smooth action of $T^{n}$ with non-empty fixed point set is called a torus manifold if a preferred orientation is given for each characteristic submanifold. A characteristic submanifold is, by definition, any codimension two closed connected submanifold of $Y$, which is fixed by some circle subgroup of $T^{n}$ and contains at least one $T^{n}$-fixed point.

Definition 2.6. [HM03] A torus manifold $M$ of dimension $2 n$ is locally standard if every point in $M$ has an invariant neighborhood $U$ weakly equivariantly diffeomorphic to an open subset $W \subset \mathbb{C}^{n}$ invariant under the standard $T^{n}$-action on $\mathbb{C}^{n}$.

We briefly recall the construction of some locally standard torus manifolds.

Definition 2.7. A function $\xi: \mathcal{F}(P) \rightarrow \mathbb{Z}^{n}$ is called a characteristic function on $P$ if it satisfies the following condition: Whenever $F=\bigcap_{j=1}^{k} F_{i_{j}}$ is an $(n-k)$-dimensional face of $P$, the span of the vectors $\xi\left(F_{i_{1}}\right), \xi\left(F_{i_{2}}\right), \ldots, \xi\left(F_{i_{k}}\right)$ is a $k$-dimensional direct summand of $\mathbb{Z}^{n}$. We will denote $\xi\left(F_{i}\right)$ by $\xi_{i}$ for simplicity and call it the characteristic vector of $F_{i}$.

For any face $F=\bigcap_{j=1}^{k} F_{i_{j}}$ of $P$, let $N(F)$ be the submodule of $\mathbb{Z}^{n}$ generated by $\left\{\xi_{i_{1}}, \ldots, \xi_{i_{k}}\right\}$. The module $N(F)$ defines a sub-torus $T_{F}$ of $T^{n}=\mathbb{Z}^{n} \otimes \mathbb{R} / \mathbb{Z}^{n}=\mathbb{R}^{n} / \mathbb{Z}^{n}$ as follows.

$$
T_{F}:=(N(F) \otimes \mathbb{R}) / N(F) .
$$

Define an equivalence relation $\sim$ on the product space $T^{n} \times P$ by

$$
(t, x) \sim(u, y) \text { if } x=y \text { and } u^{-1} t \in T_{F}
$$

where $F$ is the unique face of $P$ whose relative interior contains $x$.

We denote the quotient space as follows.

$$
M(P, \xi):=\left(T^{n} \times P\right) / \sim .
$$

The space $M(P, \xi)$ is a $2 n$-dimensional locally standard torus manifold. The proof of this is analogous to the toric manifold case in [DJ91]. The $T^{n}$ action on $\left(T^{n} \times P\right)$ induces a natural effective action of $T^{n}$ on $M(P, \xi)$, which is locally standard. Let $\pi: M(P, \xi) \rightarrow P$ be the projection or orbit map defined by $\pi([(t, x)])=x$. Since $M(P, \xi)$ is locally standard, the $T^{n}$-fixed point set corresponds bijectively to the set of vertices of $P$. Observe that the spaces $X_{i}:=\pi^{-1}\left(F_{i}\right), i=1, \ldots, m$, are the characteristic submanifolds of $M(P, \xi)$. Each $X_{i}$ is a $2(n-1)$-dimensional manifold. We say that $M(P, \xi)$ is the torus manifold derived from the characteristic pair $(P, \xi)$. 
Let $M$ be a locally standard torus manifold and $P=M / T^{n}$ be the orbit space of $T^{n}$-action on $M$. So $P$ is a manifold with corners and vertices of $P$ correspond to the $T^{n}$-fixed points of $M$. Let $\mathfrak{q}: M \rightarrow P$ be the quotient projection. Let $\mathcal{F}(P)=\left\{P_{1}, \ldots, P_{m}\right\}$ be the facets of $P$. Then the characteristic submanifolds (codimension-two submanifolds fixed pointwise by a circle subgroup of $T^{n}$ ) of $M$ are $\left\{M_{i}=\mathfrak{q}^{-1}\left(P_{i}\right): i=1, \ldots, m\right\}$. So we can choose a map

$$
\lambda: \mathcal{F}(P) \rightarrow \mathbb{Z}^{n}
$$

such that $\lambda\left(P_{i}\right)$ is the primitive vector in $\mathbb{Z}^{n}$ and determines the circle subgroup of $T^{n}$ fixing $M_{i}$.

Lemma 2.8. [MP06] If $P_{i_{1}} \cap \cdots \cap P_{i_{k}}$ is non-empty, then $\lambda\left(P_{i_{1}}\right), \ldots, \lambda\left(P_{i_{k}}\right)$ is a part of a basis of $\mathbb{Z}^{n}$.

So $\lambda$ is a characteristic function on $P$. Let $M(P, \lambda)$ be the torus manifold obtained by construction from the pair $(P, \lambda)$.

Lemma 2.9. [MP06] If $H^{2}(P, \mathbb{Z})=0$, then there is an equivariant homeomorphism $M(P, \lambda) \rightarrow M$ covering the identity on $P$.

2.3. Equivariant connected sums. In Section 6 of [GK98] equivariant connected sum of two smooth manifolds with $T^{n}$-action is discussed explicitly. Equivariant connected sum of toric manifolds is discussed in Section 6 of [BR01]. We briefly recall the argument for equivariant connected sum of torus manifolds in the following. Let $M_{1}$ and $M_{2}$ be two smooth locally standard torus manifolds of dimension $2 n$. Let $T_{1} \subset M_{1}$ and $T_{2} \subset M_{2}$ be two orbit of same dimension. Now changing the action (if necessary) of $T^{n}$ on $M_{2}$ by an automorphism of $T^{n}$, we may assume that $T_{1}$ and $T_{2}$ have same stabilizer $G$ and for which the isotropy actions are isomorphic. Then by the slice theorem each orbit $T_{i}$ has a tubular neighborhood $V_{i}$ equivariantly diffeomorphic to $T^{n} \times_{H} D^{2 \ell}$, where $D^{2 \ell}$ is a disc in a linear $G$-representation $\mathbb{R}^{2 \ell}$ for some $\ell$. Identifying the deleted neighborhood $V_{1}-T_{1}$ and $V_{2}-T_{2}$ via an orientation reversing equivariant diffeomorphism we get a smooth manifold, denoted by $M_{1} \#_{T^{k}} M_{2}$, with a natural locally standard $T^{n}$-action. If equivariant homeomorphism is considered, then we may loose smooth structure.

The orbit space of $T^{n}$-action on $M_{1} \#_{T^{k}} M_{2}$ can be described as follows. Let $\mathfrak{q}_{1}: M_{1} \rightarrow Q_{1}$ and $\mathfrak{q}_{2}: M_{2} \rightarrow Q_{2}$ be the orbit maps for $T^{n}$-action on $M_{1}$ and $M_{2}$ respectively. Let $\mathfrak{q}_{1}\left(T_{1}\right)=x_{1} \in Q_{1}$ and $\mathfrak{q}_{2}\left(T_{2}\right)=x_{2} \in Q_{2}$. So $x_{1}$ and $x_{2}$ belong to the relative interior of $k$-dimensional face $H_{1}$ and $H_{2}$ of $Q_{1}$ and $Q_{2}$ respectively. Delete a neighborhood $U_{1}$ of $x_{1}$ in $Q_{1}$ such that the closure of $U_{1}$ is homeomorphic as manifold with corners to

$$
\left\{\left(x_{0}, \ldots, x_{n}\right) \in \mathbb{R}^{n}: x_{0}^{2}+\cdots+x_{n}^{2} \leq 1 \text { and } x_{i} \geq 0 \text { if } i \geq k\right\} \text { and } \bar{U} \cap F=\emptyset
$$

for any face $F$ of $Q_{1}$ with $F \cap H_{1}^{0}=\emptyset$. Let $Q_{1}^{\prime}$ be the remaining manifold with corners. Then $Q_{1}^{\prime}$ has a new facet $\widetilde{H}_{1}$ which is homeomorphic as manifold with corners to

$$
\left\{\left(x_{0}, \ldots, x_{n}\right) \in \mathbb{R}^{n}: x_{0}^{2}+\cdots+x_{n}^{2}=1 \text { and } x_{i} \geq 0 \text { if } i \geq k\right\} .
$$


Similarly we can construct the manifold with corners $Q_{2}^{\prime}$ from $Q_{2}$. Let $H_{i}$ be a connected component of $F_{1}^{i} \cap \ldots \cap F_{n-k}^{i}$ for some unique facets $F_{1}^{i}, \ldots, F_{n-k}^{i}$ of $Q_{i}$. By assumption on the stabilizer of $T_{1}, T_{2}$, we may assume that the characteristic vector of $F_{j}^{1}$ and $F_{j}^{2}$ are same for $j=1, \ldots, n-k$. From Subsection 2.1 one can construct a manifold with corners, denoted by $Q_{1} \# x_{x_{1}, x_{2}} Q_{2}$, by gluing $Q_{1}^{\prime}$ and $Q_{2}^{\prime}$ at $x_{1}$ and $x_{2}$ such that $F_{j}^{1} \# x_{1}, x_{2} F_{j}^{2}$ makes a new facet for $j=1, \ldots, n-k$. Then $M_{1} \#_{T^{k}} M_{2}$ is a locally standard torus manifold over $Q_{1} \#_{x_{1}, x_{2}} Q_{2}$.

Example 2.10. Let $[n]=\{1, \ldots, n\}$ for all positive integer $n$. Consider

$$
S^{2 n}=\left\{\left(z_{1}, \ldots, z_{n}, x\right) \in \mathbb{C}^{n} \times \mathbb{R}:\left|z_{1}\right|^{2}+\cdots+\left|z_{n}\right|+x^{2}=1\right\} .
$$

The natural $T^{n}$-action on $S^{2 n}$ is defined by

$$
\left(\left(t_{1}, \ldots, t_{n}\right) \times\left(z_{1}, \ldots, z_{n}, x\right)\right) \rightarrow\left(t_{1} z_{1}, \ldots, t_{n} z_{n}, x\right) .
$$

This is a locally standard action and the orbit map is defined by

$$
\pi\left(z_{1}, \ldots, z_{n}, x\right) \rightarrow\left(\left|z_{1}\right|, \ldots,\left|z_{n}\right|, x\right) .
$$

So the orbit space is

$$
Q^{n}:=\left\{\left(x_{1}, \ldots, x_{n}, x\right) \in \mathbb{R}^{n+1}: x_{1}^{2}+\cdots+x_{n}^{2}+x^{2}=1 \text { and } x_{i} \geq 0 \text { for } i=1, \ldots, n\right\} .
$$

Therefore, $Q^{n}$ is a nice manifold with corners. The vertices of $Q^{n}$ are $\{(0, \ldots, 0, \pm 1)\}$. A codimension- $k(0<k<n)$ face of $Q^{n}$ is given by

$$
\left\{\left(x_{1}, \ldots, x_{n}, x\right) \in Q^{n}: x_{i_{1}}=\cdots=x_{i_{k}}=0 \text { for some }\left\{i_{1}, \ldots, i_{k}\right\} \subset[n]\right\} .
$$

In particular, the edges of $Q^{n}$ are given by $E_{i}:=\left\{\left(0, \ldots, 0, x_{i}, 0, \ldots, 0, x\right) \in Q^{n}\right\}$ for $i=1, \ldots, n$. Let $F_{i}$ be the face of $Q^{n}$ defined by

$$
F_{i}:=\left\{\left(x_{1}, \ldots, x_{i-1}, 0, x_{i+1} \ldots, x_{n}, x\right) \in Q^{n}\right\}
$$

for $i \in\{1, \ldots, n\}$. Then facets of $Q$ are $\left\{F_{1}, \ldots, F_{n}\right\}$. The subgroup which fixes $\pi^{-1}\left(F_{i}\right)$ is $\left\{\left(0, \ldots, 0, t_{i}, 0, \ldots, 0\right) \in T^{n}\right\}$ for $i=1, \ldots, n$. Let $F$ be a $k$-dimensional face of $Q$. Let $x_{1}, x_{2}$ be two points in the relative interior of $F$. Let $T_{i}=\pi^{-1}\left(x_{i}\right)$ for $i=1,2$. Then $T_{i}$ satisfy the assumption in previous connected sum construction. So we can construct locally standard torus manifold $S^{2 n} \#_{T^{k}} S^{2 n}$. We denote the inclusion $T_{p} \subset S^{2 n}$ by $\tau_{p}$ for $p=1,2$.

Example 2.11. From the equivariant connected sum construction we have that the orbit space of the torus manifold $S^{2 n} \#_{T^{k}} S^{2 n}$ is $Q^{n} \#_{x_{1}, x_{2}} Q^{n}$. The orbit spaces of $S^{6}$, $S^{6} \# T^{1} S^{6}, S^{6} \#_{T^{2}} S^{6}$ and $S^{6} \#_{T^{3}} S^{6}$ are given in Figure 2 respectively. Note that the manifold with corners in Figure $2(d)$ is homeomorphic to $\partial Q^{3} \times[0,1]$ as manifold with corners, so it is not contractible.

Lemma 2.12. Let $M$ be locally standard torus manifold with orbit space $P$. If $P$ is a connected sum of simple polytopes, then $M$ is an equivariant connected sum of toric manifolds. 


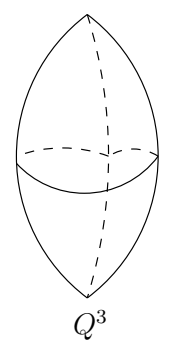

(a)

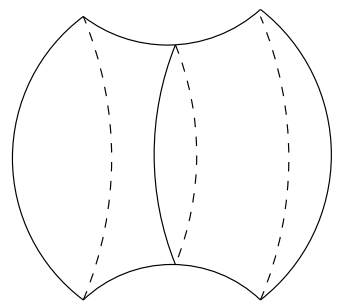

(b)

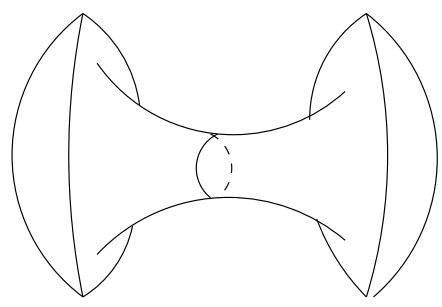

$(c)$

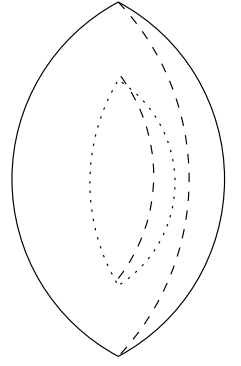

$(d)$

FiguRE 2. Some connected some of manifolds with corners.

Proof. It is enough to prove when $P$ is the connected sum $Q \#_{x_{F}, y_{G}} R$ where $x_{F}$ and $y_{G}$ are interior points of the $k$-dimensional faces $F$ and $G$ of the simple polytope $Q$ and $R$ in $\mathbb{R}^{n}$ respectively. Let $B$ and $C$ be open $n$-ball around $x_{F}$ and $y_{G}$ respectively in $\mathbb{R}^{n}$ such that conditions in 2.1 are satisfied. So there are diffeomorphisms

$$
f: Q-B \rightarrow Q \#_{x_{F}, y_{G}} R \text { and } g: R-C \rightarrow Q \#_{x_{F}, y_{G}} R
$$

onto its image. Let $\left\{P_{1}, \ldots, P_{m}\right\},\left\{Q_{1}, \ldots, Q_{k}\right\}$ and $\left\{R_{1}, \ldots, R_{l}\right\}$ be the facets of $P, Q$ and $R$ respectively. Let $\xi:\left\{P_{1}, \ldots, P_{m}\right\} \rightarrow \mathbb{Z}^{n}$ be the characteristic function of the torus manifold $M$. We define a function

$$
\eta_{1}:\left\{Q_{1}, \ldots, Q_{k}\right\} \rightarrow \mathbb{Z}^{n} \text { and } \eta_{2}:\left\{R_{1}, \ldots, R_{l}\right\} \rightarrow \mathbb{Z}^{n}
$$

by

$$
\eta_{1}(F)=\xi\left(P_{i}\right) \text { if } f(F)=P_{i} \cap f(Q) \text { and } \eta_{2}(F)=\xi\left(P_{j}\right) \text { if } g(F)=P_{j} \cap g(R)
$$

respectively. So $\eta_{1}$ and $\eta_{2}$ are characteristic function of $Q$ and $R$ respectively. Let $M_{1}$ and $M_{2}$ be the toric manifolds over $Q$ and $R$ corresponding to these characteristic functions respectively. Let $q: M_{1} \rightarrow Q$ and $r: M_{2} \rightarrow R$ be the orbit maps. Let $\hat{B}=q^{-1}(B)$ and $\hat{C}=r^{-1}(C)$. Let $M_{1} \#_{x_{F}, y_{G}} M_{2}$ be the space obtained by identifying the boundary of $M_{1}-\hat{B}$ and $M_{2}-\hat{C}$ via an equivariant diffeomorphism. Then $M$ is equivariantly homeomorphic to $M_{1} \#_{x_{F}, y_{G}} M_{2}$.

Lemma 2.13. Let $M$ and $N$ be $2 n$-dimensional toric manifolds. Then $M \#_{T^{k}} N$ is homeomorphic to $M \# N \#\left(S^{2 n} \#_{T^{k}} S^{2 n}\right)$.

Proof. Let $\pi_{1}: M \rightarrow P_{1}$ and $\pi_{2}: N \rightarrow P_{2}$ be two orbit maps. Let $\iota_{1}: T^{k} \rightarrow M$ and $\iota_{2}: T^{k} \rightarrow N$ be embedding of $k$-dimensional orbits. So $\operatorname{Im}\left(\iota_{1}\right)$ and $\operatorname{Im}\left(\iota_{2}\right)$ are interior point of $k$-dimensional face $F$ and $G$ of $P_{1}$ and $P_{2}$ respectively. Let $v_{1}$ and $v_{2}$ be vertices of $F$ and $G$ respectively. So $v_{1}$ and $v_{2}$ are vertices of $P_{1}$ and $P_{2}$ respectively.

We may assume the polytopes $P_{1}$ and $P_{2}$ are subset of $\mathbb{R}^{n}$. Let $B_{i}$ be an open ball in $\mathbb{R}^{n}$ with center $v_{i}$ such that $\overline{B_{i} \cap P_{i}} \cap F$ is empty for any face not containing the vertex $v_{i}$ for $i=1,2$. Let $U_{i}=B_{i} \cap P_{i}$ for $i=1,2$. So $\pi_{i}^{-1}\left(U_{i}\right)$ is equivariantly homeomorphic to $\mathbb{C}^{n}$. Without loss of generality, we may consider that $\operatorname{Im}\left(\iota_{i}\right)$ belongs to $\pi_{i}^{-1}\left(U_{i}\right)$ for $i=1,2$. So $M$ and $N$ are equivariantly homeomorphic to 
$M \#_{v_{1}} S^{2 n}$ and $N \#_{v_{2}} S^{2 n}$ respectively such that $\operatorname{Im}\left(\iota_{1}\right)$ and $\operatorname{Im}\left(\iota_{2}\right)$ are $k$-dimensional orbit in $S^{2 n}$.

Since $S^{2 n}$ is a torus manifold, we can construct $S^{2 n} \#_{T^{k}} S^{2 n}$. So $M \#_{T^{k}} N$ is equivariantly homeomorphic to $M \#_{v_{1}}\left(S^{2 n} \#_{T^{k}} S^{2 n}\right) \#_{v_{2}} N$. Forgetting the equivariantness of the connected sum $M \#_{v_{1}} S^{2 n}$ and $N \#_{v_{2}} S^{2 n}$, we get that $M \#_{T^{k}} N$ is homeomorphic to $M \# N \#\left(S^{2 n} \#_{T^{k}} S^{2 n}\right)$.

Corollary 2.14. Let $M_{1}, \ldots, M_{n}$ be $2 n$-dimensional toric manifolds. Then

$$
M_{1} \#_{T^{k_{1}}} M_{2} \#_{T^{k_{2}}} \cdots \#_{T^{k_{n-1}}} M_{n}
$$

is homeomorphic to

$$
M_{1} \# \cdots \# M_{n} \#\left(S^{2 n} \#_{T^{k_{1}}} S^{2 n}\right) \# \cdots \#\left(S^{2 n} \#_{T^{k_{n-1}}} S^{2 n}\right) .
$$

Proof. This follows from the successive application of Lemma 2.13.

\section{TORUS COMPLEMENT OF EVEN DIMENSIONAL SPHERES}

In this section we discuss the homotopy type of the complement of an orbit of the torus action on even dimensional spheres. We show that if $T^{k} \subset S^{2 n}$ is an orbit then $S^{2 n} \backslash T^{k}$ is homotopic to wedge of some lower dimensional spheres. Let $[n]=\{1, \ldots, n\}$ for all positive integer $n$. and $\mathcal{L}(n, 0)$ the set of faces of $Q^{n}$ including $Q^{n}$. For $1 \leq k \leq n$, let

$$
\mathcal{L}(n, k)=\mathcal{L}(n, 0)-\left\{F \in \mathcal{L}(n, 0): F \text { contains the edges }\left\{E_{k}, \ldots, E_{n}\right\}\right\}
$$

for $k=1, \ldots, n$. Let

$$
V([n], k):=\bigcup_{F \in \mathcal{L}(n, k)} F \text { and } U([n], k):=\pi^{-1}(V([n], k))
$$

for $k=0,1, \ldots, n$. The following Proposition true from the definition.

Proposition 3.1. $V([n], n)=V([n-1])$ and $U([n], n)=U([n-1])$ for all $n \geq 2$.

Let $Q_{k}^{n-1}$ be the facet of $Q^{n}$ not containing the edge $E_{k}$ for $k \in\{1, \ldots, n\}$. Define $V([n]-\{k-1\})=Q_{k-1}^{n-1}$ for $k \in\{2, \ldots n\}$. Then similarly as above we can define $V([n]-\{k-1\}, k)$ and $U([n]-\{k-1\}, k)$ for $k \in\{2, \ldots, n\}$.

Proposition 3.2. For all $k, 2 \leq k \leq n$ we have the following homotopy push-out diagram.

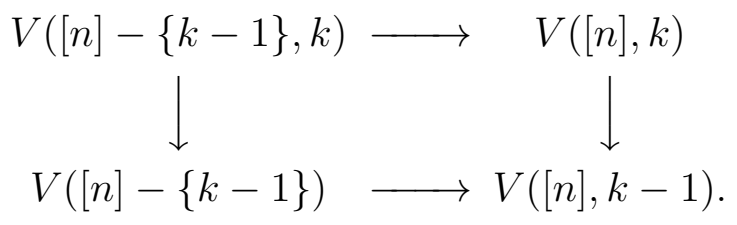


and

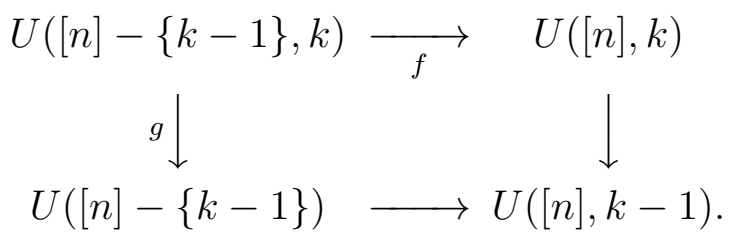

Moreover, $f$ and $g$ are null homotopic.

Proof. The maps in both the diagram in the Proposition 3.2 are natural. We'll show $f$ and $g$ are null homotopic. Since $U([n]-\{k-1\})$ is a sphere of dimension $2 n-2$, the map $g$ is null homotopic by dimension reason.

First we want to define a homotopy from $V([n]-\{k-1\}, k)$ to $E_{k-1}$ relative to the vertices of $Q^{n}$. Let $z=\left(x_{1}, \ldots, x_{k-2}, 0, x_{k}, \ldots, x_{n}, x\right) \in Q^{n}$. Then we have a continuous onto map $h: V([n]-\{k-1\}, k) \rightarrow E_{k-1}$ defined by

$$
\left(x_{1}, \ldots, x_{k-2}, 0, x_{k}, \ldots, x_{n}, x\right) \rightarrow\left(0, \ldots, 0, x_{k-1}, 0, \ldots, 0, x\right) .
$$

Hence the map $H: V([n]-\{k-1\}, k) \times[0,1] \rightarrow Q^{n}$ defined by

$$
H(z, r)=\frac{(1-r) z+r h(z)}{\|(1-r) z+r h(z)\|}
$$

gives a homotopy from $V([n]-\{k-1\}, k)$ to $E_{k-1}$ relative to their vertices. Let $F_{k-1}=\left\{\left(x_{1}, \ldots, x_{k-2}, 0, x_{k}, \ldots, x_{n}, x\right) \in Q^{n}\right\}$. Then the isotropy group, denote it by $T_{k-1}$, of $\pi^{-1}\left(F_{k-1}\right)$ is the $(k-1)$-th circle subgroup of $T^{n}$. Let

$$
T_{k-1}^{n-1}:=T^{n} / T_{k-1}=\left\{\left(t_{1}, \ldots, t_{k-2}, 1, t_{k}, \ldots, t_{n}\right) \in T^{n}\right\} .
$$

Note that

$$
\left(T^{n} \times V([n]-\{k-1\}, k)\right) / \sim=\left(T_{k-1}^{n-1} \times V([n]-\{k-1\}, k)\right) / \sim
$$

where the equivalence relation $\sim$ is defined in (2.3). The homotopy $H$ induces a map

defined by

$$
\widehat{H}: T_{k-1}^{n-1} \times V([n]-\{k-1\}, k) \times[0,1] \rightarrow T^{n} \times Q^{n},
$$

$$
\left(\left(t_{1}, \ldots, t_{k-2}, 1, t_{k}, \ldots, t_{n}\right), z, r\right) \rightarrow\left(\left(t_{1}, \ldots, t_{k-2}, 1, t_{k}, \ldots, t_{n}\right), H(z, r)\right) .
$$

Observe that the map $\widehat{H}$ induces a map, denoted by $\bar{H}$, from

$$
\left(T_{k-1}^{n-1} \times V([n]-\{k-1\}, k)\right) / \sim \times[0,1] \rightarrow\left(T^{n} \times Q^{n}\right) / \sim .
$$

Now

$$
\bar{H}\left(\left(t_{1}, \ldots, t_{k-2}, 1, t_{k}, \ldots, t_{n}\right), z, 1\right)=\left(\left(t_{1}, \ldots, t_{k-2}, 1, t_{k}, t_{n}\right), h(z)\right) / \sim=h(z) .
$$

So $\bar{H}$ is a homotopy from $\left(T^{n} \times V([n]-\{k-1\}, k)\right) / \sim$ to $\pi^{-1}\left(E_{k-1}\right) \cong S^{2}$. Since the image of $h$ is homeomorphic to $[0,1]$, the map $f$ is null homotopic.

Lemma 3.3. The space $U([n], k)$ is strong deformation retract of $S^{2 n}-T^{n-k+1}$ for $1 \leq k \leq n$ and $U([n]-\{k-1\}, k)$ is strong deformation retract of $S^{2 n-2}-T^{n-k+1}$ for $2 \leq k \leq n$. 
Proof. Let $\bar{\pi}: Q^{n} \rightarrow \mathbb{R}^{n+1}$ be the map defined by

$$
\bar{\pi}\left(\left(x_{1}, \ldots, x_{n}, x\right)\right)= \begin{cases}\left(x_{1}^{2}, \ldots, x_{n}^{2}, \frac{x^{3}}{|x|}\right) & \text { if } x \neq 0 \\ \left(x_{1}^{2}, \ldots, x_{n}^{2}, 0\right) & \text { if } x=0\end{cases}
$$

Let $P^{n}$ be the image of $\bar{\pi}$. So $\bar{\pi}: Q^{n} \rightarrow P^{n}$ is a homeomorphism. Let $\xi: S^{2 n} \rightarrow$ $P^{n}$ be the composition of $\pi$ and $\bar{\pi}$. Let $F_{k}=\left\{\left(0, \ldots, 0, y_{k}, \ldots, y_{n}, 0\right) \in P^{n}\right\}$ for $k \in\{1, \ldots, n\}$. Let $z$ be an interior point of $F_{k}$. Then $\xi^{-1}(z) \cong T^{n-k+1}$. So $S^{2 n}-T^{n-k+1}=S^{2 n}-\xi^{-1}(z)$. Let $x \in P^{n}-\{z\}$ and $\ell(z, x)$ be the line in $\mathbb{R}^{n+1}$ passing through the points $\{z, x\}$. From the definition of $V([n], k)$ we can see that $\ell(z, x)$ intersect $\bar{\pi}(V([n], k))$ in a unique point, say $f_{z}(x)$. So this induces a continuous map $f_{z}: P^{n}-\{z\} \rightarrow \bar{\pi}(V([n], k))$. We define a homotopy

$$
H: P^{n}-\{z\} \times[0,1] \rightarrow P^{n}-\{z\}
$$

by $H(x, r)=(1-r) x+r f_{z}(x)$. Note that $H$ fixes $\bar{\pi}(V([n], k))$. So

$$
\bar{H}:=H \circ(\bar{\pi} \times I d)=Q^{n}-\bar{\pi}^{-1}(z) \times[0,1] \rightarrow Q^{n}-\bar{\pi}^{-1}(z)
$$

is a homotopy from $Q^{n}-\bar{\pi}^{-1}(z)$ to $V([n], k)$. If $\bar{\pi}^{-1}(x)$ belongs to the relative interior of a face $F$ of $Q^{n}$ then $\bar{\pi}^{-1}\left(f_{z}(x)\right)$ belongs to the relative interior of a face of $F$. So $T_{F(x)}$ is a subgroup of $T_{F(H(x, r))}$ for every $r \in[0,1]$. Hence $\bar{H}$ induces a homotopy from $\left(T^{n} \times Q^{n}-\bar{\pi}^{-1}(z)\right) / \sim$ to $\left(T^{n} \times V([n], k)\right) / \sim$ defined by

$$
\left([t, x]^{\sim}, r\right) \rightarrow[t, \bar{H}(x, r)]^{\sim}
$$

This proves the first statement of Lemma. The second statement can be proved similarly.

Lemma 3.4. Let $t=n+1-k$. Then $U([n], k) \simeq \vee_{i=1}^{t} \vee_{\left(\begin{array}{c}t \\ i\end{array}\right)} S^{2 n-1-i}$.

Proof. Clearly Lemma hold for $n=1$ and $k=1$. Now assume that Lemma holds for all $U(j, k)$ such that $j \leq n-1$ and for $j \geq k$. So by this assumption

$$
U([n]-\{k-1\}, k) \simeq \vee_{i=1}^{t} \vee_{\left(\begin{array}{c}
t \\
i
\end{array}\right)} S^{2 n-3-i},
$$

where $t=n-k+1$. For $k=n, t=1$ and $U([n], k) \simeq S^{2 n}-S^{1} \simeq S^{2 n-2}$. So Lemma 3.4 holds. Using the induction hypothesis and Proposition 3.2 we have the following homotopy push-out.

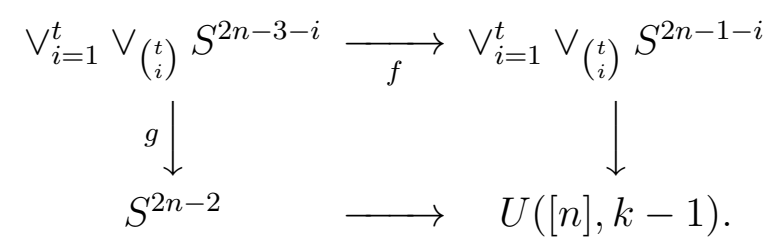


Since $f$ and $g$ are null homotopic,

$$
\begin{aligned}
U([n], k-1) & \simeq \vee_{i=1}^{t} \vee_{\left(\begin{array}{c}
t \\
i
\end{array}\right)} S^{2 n-1-i} \vee \vee_{i=1}^{t} \vee_{\left(\begin{array}{c}
t \\
i
\end{array}\right)} S^{2 n-2-i} \vee S^{2 n-2} \\
& \simeq \vee_{i=1}^{t} \vee_{\left(\begin{array}{c}
t \\
i
\end{array}\right)} S^{2 n-1-i} \vee \vee_{i=0}^{t} \vee_{\left(\begin{array}{c}
t \\
i
\end{array}\right)} S^{2 n-2-i} \\
& \simeq \vee_{i=1}^{t} \vee_{\left(\begin{array}{c}
t+1 \\
i
\end{array}\right)} S^{2 n-1-i}, \text { since }\left(\begin{array}{c}
t \\
i
\end{array}\right)+\left(\begin{array}{c}
t \\
i-1
\end{array}\right)=\left(\begin{array}{c}
t+1 \\
i
\end{array}\right) \text { for } i \geq 1 .
\end{aligned}
$$

Which shows that Lemma 3.4 holds for $U([n], k-1)$.

\section{Homology GROUPS OF CERTAIN TORUS MANIFOLDS}

In this section we compute the homology groups of torus manifolds which are equivariant connected sum of toric manifolds. First we compute the homology of $S^{2 n} \#_{T^{k}} S^{2 n}$ of Example 2.10 where $1 \leq k \leq n$. Other cases can be computed using Corollary 2.14. We adhere the notations of previous sections.

Proposition 4.1. Let $n>2, k^{\prime}=\min \{k, n-3\}, q(k)=1$ if $k=n$, and $q(k)=0$ if $k=n-2, n-1$. Then homology groups of $S^{2 n} \#_{T^{k}} S^{2 n}$ are given by the following.

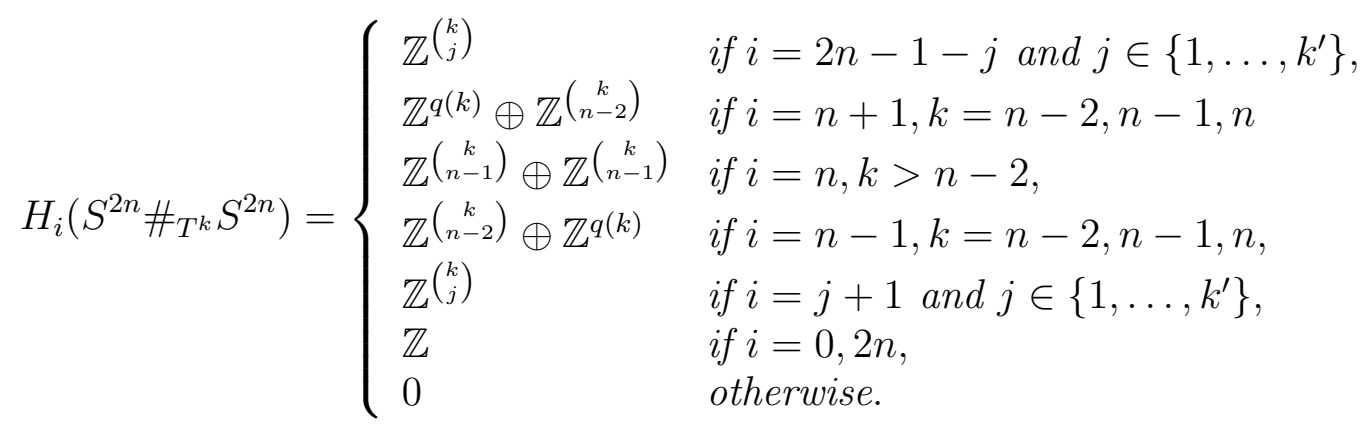

Proof. Let the orbit $T^{k} \hookrightarrow S^{2 n}$ be $\pi^{-1}(x)$ such that $x$ belongs to the relative interior $F^{0}$ of a $k$-dimensional face $F$ of $Q^{n}$. Without any loss, we may assume the edges of $F$ are $E_{n-k+1}, \ldots, E_{n}$ of $Q^{n}$.

Let $\alpha:[-1,1] \rightarrow Q^{n}$ be a simple path in $Q^{n}$ defined by

$$
\alpha(t)= \begin{cases}\frac{(1+t) x-t(0, \ldots, 0,-1)}{\|(1+t) x-t(0, \ldots, 0,-1)\|} & \text { if } t \in[-1,0], \\ \frac{t x+(1-t)(0, \ldots, 0,1)}{\|t x+(1-t)(0, \ldots, 0,1)\|} & \text { if } t \in[0,1] .\end{cases}
$$

joining the points $(0, \ldots, 0,-1)$ and $(0, \ldots, 0,1)$, and passing through $x$. Note that $\alpha((0,1)) \subseteq F^{0}$, see Figure 3 for examples when $n=3$. From the equivariant connected sum construction in subsection 2.3, we have the orbit space of $S^{2 n} \#_{T^{k}} S^{2 n}$ is $Q^{n} \#_{x, x} Q^{n}$ and the corresponding orbit map given by

$$
\pi: S^{2 n} \#_{T^{k}} S^{2 n} \rightarrow Q^{n} \#_{x, x} Q^{n} .
$$

For simplicity we assume $Q_{i}=Q^{n}$ represents the $i$-th component in $Q^{n} \#_{x, x} Q^{n}$. We may perform the connected sum such that

$$
\alpha([-1,1]) \#_{x, x} \alpha([-1,1])=\alpha_{1}([-1,1]) \sqcup \alpha([-1,1]) \subset Q^{n} \#_{x, x} Q^{n}
$$


such that $\alpha_{i}:[-1,1] \rightarrow Q^{n} \#{ }_{x, x} Q^{n}$ satisfies $\alpha_{i}(0)=\alpha(-1) \in Q_{i}, \alpha_{i}(1)=\alpha(1) \in Q_{i}$ and $\alpha_{i}(0,1) \subseteq\left(F \#_{x, x} F\right)^{0} \subset Q^{n} \#_{x, x} Q^{n}$ for $i=1,2$.

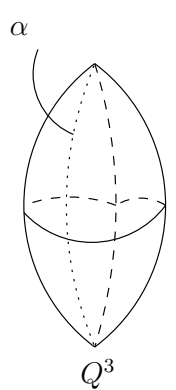

(a)

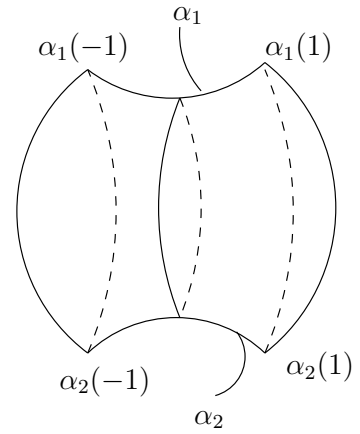

(b)

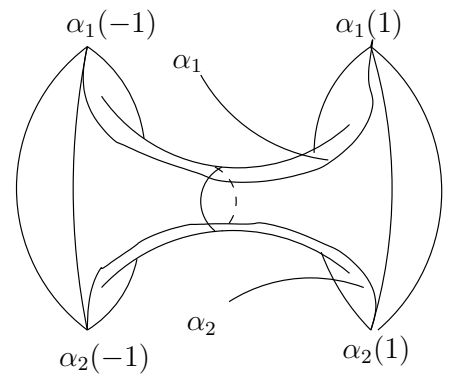

(c)

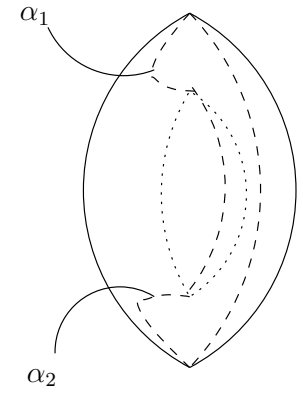

$(d)$

Figure 3. The paths $\alpha, \alpha_{1}$ and $\alpha_{2}$ is some examples.

Recall from Section 3 that there is a face preserving strong deformation from $Q^{n}-\{x\}$ onto $V([n], n-k+1) \subset Q^{n}$. We may assume $V([n], n-k+1)$ is a subset of $Q_{2}$. Therefore from the connected sum $Q^{n} \#_{x, x} Q^{n}$, we have that the complement of

$$
D:=\alpha_{2}([-1,1]) \cup V([n], n-k+1) \subset Q^{n} \#_{x, x} Q^{n}
$$

is homeomorphic to $\mathbb{R}_{\geq 0}^{n}$ as manifold with corners. Let $B=Q^{n} \#_{x, x} Q^{n} \backslash D$ and $C=Q^{n} \#_{x, x} Q^{n} \backslash\left\{\alpha_{1}(-1)\right\}$. Then following the arguments in Section 3, one can show that there is a face preserving strong deformation from $B \backslash\left\{\alpha_{1}(-1)\right\}$ onto a vertex cut at $\alpha_{1}(-1)$ in $Q^{n} \#_{x, x} Q^{n}$. Here the vertex cut at $\alpha_{1}(-1)$ in $Q^{n} \#_{x, x} Q^{n}$ is homeomorphic to $\Delta^{n-1}$ as manifold with corners. So $\pi^{-1}\left(B \backslash\left\{\alpha_{1}(-1)\right\}\right)$ is homotopic to $S^{2 n-1}$.

Also $D$ is deformation retract of $C$ in $Q^{n} \#{ }_{x, x} Q^{n}$ by a face preserving homotopy, and hence $\pi^{-1}(D)$ is a deformation retract of $\pi^{-1}\left(D_{n}\right)$. Therefore,

$$
\pi^{-1}(C) \cap \pi^{-1}\left(D_{n}\right) \simeq S^{2 n-1} .
$$

We have $\alpha_{i}(-1,1) \subset\left(F \#_{x, x} F\right)^{0}$. So $\pi^{-1}\left(\alpha_{2}([-1,1])\right)$ is homeomorphic to the suspension $\Sigma T^{k}$, since the image $\alpha((0,1))$ belongs to the interior of a $k$ dimensional face $F$. Then

$$
\pi^{-1}(D)=\Sigma T^{k} \vee U([n], n-k+1)
$$

By Lemma 3.4,

$$
U([n], n-k+1) \simeq \vee_{i=1}^{k} \vee_{\left(\begin{array}{c}
k \\
i
\end{array}\right)} S^{2 n-1-i} .
$$

Note that dimension of $U([n], n-k+1)$ is $2 n-2$. Then using Mayer-Vietoris sequence for the open subsets $\pi^{-1}(B)$ and $\pi^{-1}(C)$ of $S^{2 n} \#_{T^{k}} S^{2 n}$, we get the lemma.

Following the notation and proof of Proposition 4.1, we get the following when $n=2$. 
Proposition 4.2. For $k \in\{1,2\}$, the homology groups of $S^{4} \#_{T^{k}} S^{4}$ are given by the following.

$$
H_{i}\left(S^{4} \#_{T^{k}} S^{4}\right)= \begin{cases}\mathbb{Z}^{k-1} & \text { if } i=1,3, k=1,2, \\ \mathbb{Z}^{2} & \text { if } i=2, \\ \mathbb{Z} & \text { if } i=0,4, \\ 0 & \text { otherwise. }\end{cases}
$$

\section{Cohomology Ring of Certain torus manifolds}

In [LS05], the authors discuss the rational homotopy type of the complement of a Poincare embedding when the codimension is at least 3. We adhere the notations of previous Sections. We know that $S^{2 n}$ is a torus manifold with orbit space $Q^{n}$. Let

$$
\tau_{p}: T^{k} \rightarrow S^{2 n}
$$

be embedding of $k$-dimensional orbit for $p=1,2$ such that $\operatorname{Im}\left(\tau_{1}\right) \cap \operatorname{Im}\left(\tau_{2}\right)$ is empty. We want to find a model for $S^{2 n} \#_{T^{k}} S^{2 n}$. Note that connected sum depends on the identification of the boundary of normal neighborhood. Here we are considering the identification via identity map (possibly with reverse orientation). In this section, we assume $\mathbb{K}$ is a field of characteristic zero and $p \in\{1,2\}$.

First, we compute a model for the embedding $\tau_{p}: T^{k} \rightarrow S^{2 n}$. Let $N_{p}$ be a tubular neighborhood of $T^{k}$ in $S^{2 n}$. Then the following diagram commutes with codimension of $N_{p}$ is zero.

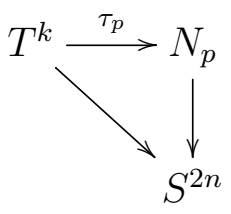

So we have the inclusion $\partial N_{p} \rightarrow \overline{S^{2 n}-N_{p}}$. This induces a map $\phi: B_{p} \rightarrow C_{p}$ from a model $B_{p}$ of $\overline{S^{2 n}-N_{p}}$ to a model $C_{p}$ of $\partial N_{p}$. Since $T^{k}$ is a $k$-dimensional orbit, its tubular neighborhood $N_{p}$ is diffeomorphic to the normal bundle of $T^{k}$ in $S^{2 n}$ which we know is trivial. So $\partial N_{p}$ is homeomorphic to $T^{k} \times S^{2 n-k-1}$. Now a model for $T^{k}$ is $\Lambda\left(a_{1}, \ldots, a_{k}: d a_{j}=0\right)$. A model for $S^{2 n-k-1}$ is $\Lambda(b: d b=0)$, where $|b|=2 n-k-1$. So we get

Proposition 5.1. A model for $\partial N_{p}$ is

$$
C=\Lambda\left(a_{1}, \ldots, a_{k}: d a_{j}=0\right) \otimes \Lambda(b: d b=0) \cong \Lambda\left(a_{1}, \ldots, a_{k}, b: d=0\right) .
$$

Proposition 5.2. A model for $\overline{S^{2 n}-N_{p}}$ is

$$
B=\mathbb{Q} 1 \oplus \mathbb{Q} c \oplus b^{\prime} \cdot \Lambda\left(a_{1}, \ldots, a_{k}\right)
$$

where $\left|a_{i}\right|=1,|c|=2 n,\left|b^{\prime}\right|=2 n-k-1$ and $d\left(b^{\prime} a_{1} \ldots a_{k}\right)=c$, for all other basis elements $d=0$.

Proof. By [LS05, Theorem 1.6] we get that as a $\Lambda(c)$-module the model of $\overline{S^{2 n}-N_{p}}$ in the proposition is correct. Now by results from Section 3, the space $\overline{S^{2 n}-N_{p}}$ is a wedge of spheres and so $\left(b^{\prime}\right)^{2}=0$. All other products in $B$ are zero for dimension reason. Therefore the result follows. 
Lemma 5.3. Let $A$ be a CDGA with trivial product structure and $\Lambda V \rightarrow A$ be a model of $A$. Let $f, g: \Lambda V \rightarrow B$ be $C D G A$ maps where $B$ has zero differential such that $H^{i}(f)$ and $H^{i}(g)$ are surjective for $2 \operatorname{con}(A)<i$. Then there exists $\phi: \Lambda V \rightarrow \Lambda V$ such that $f \phi \simeq g$.

Proof. Since product in $A$ is trivial, if necessary, one may first consider an automorphism of $A$ so that $H_{i}(f)=H_{i}(g)$. Let $\left\{a_{\alpha}\right\}$ be a graded basis of $\oplus H^{*}(A)$ for $* \leq \operatorname{dim} B$. Then there is a minimal model $\phi: \Lambda V \rightarrow A$ with $V=\left\langle a_{\alpha}, b_{j}\right\rangle$ such that $d a_{\alpha}=0,\left|b_{j}\right|>2 \operatorname{con}(A)$ and $d b_{j} \in \Lambda^{\geq 2} V$. Suppose we have already defined $\phi_{n}: \Lambda V \leq n \rightarrow \Lambda V$ such that $f \phi_{n}=\left.g\right|_{\Lambda V \leq n}$.

Let $\phi_{n+1}^{\prime}$ be any extension of $\phi_{n}$ (this always exists since all cycles in $\Lambda^{\geq 2} V$ are boundaries).

Let $\left\{v_{i}\right\}$ be a basis of $V^{n+1}$. Then $x_{i}:=\left(f \phi_{n+1}^{\prime}-g\right)\left(v_{i}\right) \in H^{n+1} B$. Therefore,

$$
x_{i}=f\left(\Sigma_{j} c_{j} a_{\alpha_{j}}\right) .
$$

Define $\phi_{n+1}\left(v_{i}\right)=\phi_{n+1}^{\prime}\left(v_{i}\right)-\Sigma_{j} c_{j} a_{\alpha_{j}}$. Then $f \phi_{n+1}=\left.g\right|_{\Lambda V \leq n+1}$. Continuing in this way we get the required map $\phi$.

Corollary 5.4. Let $f: X \rightarrow Y$ be a map such that $H^{n}(f ; \mathbb{Q})$ is a surjection for $n>2$ con $Y$. Suppose that $Y$ is rationally a wedge of spheres, $A$ is a model of $Y, B$ is a model of $X$ and $\phi: A \rightarrow B$ is a CDGA map such that $H(\phi)=H^{*}(f)$. Then $\phi$ is a model of $f$.

Proposition 5.5. A model for the inclusion $\iota: \partial N_{p} \rightarrow \overline{S^{2 n}-N_{p}}$ is $\phi: B \rightarrow C$ determined by $1 \rightarrow 1, c \rightarrow 0$ and $b^{\prime} \alpha \rightarrow$ b $\alpha$ for $\alpha \in \Lambda\left(a_{1}, \ldots, a_{k}\right)$.

Proof. First we know that $\phi$ is a CDGA map, and also by [LS05, Theorem 1.6] $\phi$ is a model of the map $\iota$ as $\Lambda(c)$-modules. Thus it induces the correct map on cohomology. Then Corrollary 5.4 implies that it is a model for the inclusion $\iota$.

Now, we compute some model for $S^{2 n} \#_{T^{k}} S^{2 n}$. Consider the subvector space $\mathcal{I} \subset$ $\Lambda\left(a_{i}, d a_{i}\right)$ with basis

$$
\left\{a_{i_{1}} \cdots a_{i_{j}} d a_{s_{1}} \cdots d a_{s_{k}} \mid k \geq 2 \text { and if } k=1 \exists t \in\{1, \ldots, j\} \text { with } i_{t} \leq s_{1}\right\} .
$$

Lemma 5.6. Then $\mathcal{I}$ is an acyclic differential ideal.

Proof. It is clear that $\mathcal{I}$ is a differential ideal. Since $\Lambda\left(a_{i}, d a_{i}\right)$ is acyclic, it is equivalent to show $E^{\prime}:=\Lambda\left(a_{i}, d a_{i}\right) / \mathcal{I}$ is acyclic. $E^{\prime}$ has a basis for representative

$$
a_{i_{1}} \cdots a_{i_{j}} \text { and }\left(d a_{i_{1}}\right) a_{i_{2}} \cdots a_{i_{j}} \text { with } i_{1} \leq \cdots \leq i_{j} .
$$

Now $d\left(a_{i_{1}} \cdots a_{i_{j}}\right)=\left(d a_{i_{1}}\right) a_{i_{2}} \cdots a_{i_{j}}$ in $E^{\prime}$. So $E^{\prime}$ is clearly acyclic.

Note that the map $\phi$ in Proposition 5.5 is not surjective. So we replace $\phi: B \rightarrow C$ with

$$
\phi^{\prime}: E^{\prime} \otimes B \rightarrow \Lambda\left(a_{i}, b\right)=C .
$$

determined by

$$
a_{i} \rightarrow a_{i}, d a_{i} \rightarrow 0, c \rightarrow 0, \text { and }\left.\phi^{\prime}\right|_{B}=\phi
$$


Let $B^{\prime}:=E^{\prime} \otimes B$. Then $\phi^{\prime}: B^{\prime} \rightarrow C$ is a surjective model for $\iota: \partial N_{p} \rightarrow \overline{S^{2 n} \backslash N_{p}}$ so that

$$
a_{i} \rightarrow a_{i}, d a_{i} \rightarrow 0 \text { and } b^{\prime} a_{i_{1}} \cdots a_{i_{\ell}} \rightarrow b a_{i_{1}} \cdots a_{i_{\ell}}
$$

for $\left\{i_{1}, \ldots, i_{\ell}\right\} \subseteq\{1, \ldots, k\}$. Then the homotopy pull back of

$$
B^{\prime}
$$

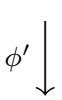

$$
B \stackrel{\phi}{\longrightarrow} C
$$

is $\mathcal{D}=\operatorname{ker}\left(\phi^{\prime}-\phi\right)$, where $\phi^{\prime}-\phi: B^{\prime} \oplus_{\mathbb{Q}} B \rightarrow C$. Let $a_{I}=a_{i_{1}} \cdots a_{i_{\ell}}$ and $\alpha_{I}=$ $\alpha_{i_{1}} \cdots \alpha_{i_{\ell}}$ for $I=\left\{i_{1}, \ldots, i_{\ell}\right\} \subseteq\{1, \ldots, k\}$ with ordering on it. Observe that $\mathcal{D}$ is generated by the elements of the form as graded module over $\mathbb{Q}$

$\left\{\left(d a_{I^{\prime}}, 0\right),(c, 0),(0, c),\left(a_{I} \otimes b^{\prime},(-1)^{|I|} b^{\prime} a_{I}\right),\left(1 \otimes b^{\prime} a_{I}, b^{\prime} a_{I}\right),\left(a_{I} \otimes b^{\prime}+\operatorname{sign}(\rho) a_{J} \otimes b^{\prime} a_{J^{\prime}}, 0\right)\right\}$

where $\rho$ is the permutation from $I$ to $J \sqcup J^{\prime}$, and $I, I^{\prime} J, J^{\prime} \subseteq\{1, \ldots, k\}$ with $I^{\prime} \neq \emptyset$.

Therefore from well-known facts, we get the following.

Proposition 5.7. $\mathcal{D}$ is a model for $S^{2 n} \#_{T^{k}} S^{2 n}$.

Let $I \subseteq\{1, \ldots, k\}$ and $I^{c}=\{1, \ldots, k\}-I$. Instead of computing $\mathcal{D}$ carefully, we construct a map $\xi: A \rightarrow \mathcal{D}$ that is a quasi-isomorphism. We define $\xi$ by

$$
s^{-1} \alpha_{i_{1}} \cdots \alpha_{i_{\ell}} \rightarrow\left(d a_{i_{1}} a_{i_{2}} \cdots a_{i_{\ell}}, 0\right), 1 \rightarrow 1, s^{-2 n+1} \alpha_{I}^{\#} \rightarrow\left(b^{\prime} a_{I^{c}}, b^{\prime} a_{I^{c}}\right), \mu_{2 n} \rightarrow(0, c),
$$

where $I \neq \emptyset$. This may not define an algebra map. So we need to replace the image of $\xi$ by a quasi-isomorphic CDGA, possibly by a quotient $\mathcal{D} / \mathcal{J}$ for some acyclic ideal $\mathcal{J}$.

Let $\mathcal{J}$ be the ideal of $\mathcal{D}$ generated by the following.

$$
\begin{aligned}
& \left\{\alpha \in \mathcal{D}^{2 n}: \alpha=(c, c) \text { or }\left(d a_{I} \otimes b^{\prime} a_{I^{c}}-c, 0\right)\right\} \cup \\
& \left\{\alpha \in \mathcal{D}^{2 n-1} \mid \alpha=\left(a_{\{1, \ldots, k\}} \otimes b^{\prime},(-1)^{k} b^{\prime} a_{\{1, \ldots k\}}\right) \text { or }\left(a_{I} \otimes b^{\prime} a_{I^{c}}+\operatorname{sign}(\rho) b^{\prime} a_{\{1, \ldots, k\}}, 0\right)\right\} \\
& \cup\left\{\left(d a_{I} \otimes b^{\prime} a_{J}, 0\right),\left(a_{I} \otimes b^{\prime} a_{J},(-1)^{|I|} b^{\prime} a_{I} a_{J}\right): \text { unless } I=J^{c}, I \neq \emptyset\right\} \cup \\
& \left\{\left(d a_{I^{\prime}} \otimes c, 0\right),\left(d a_{I^{\prime}} \otimes b^{\prime} a_{\{1, \ldots, k\}}, 0\right): I^{\prime} \neq \emptyset\right\}
\end{aligned}
$$

where $\rho$ is a permutation of $I^{c} \cup I=\{1, \ldots, k\}$.

Proposition 5.8. Let $\mathcal{J}^{\prime}$ be the submodule of $\mathcal{D}$ spanned by the elements in (5.6). Then $\mathcal{J}=\mathcal{J}^{\prime}$. In particular, the elements in (5.6) is a basis of $\mathcal{J}$.

Proof. We need to show that $\mathcal{J} \subseteq \mathcal{J}^{\prime}$, that is equivalent to show $x \cdot y \in \mathcal{J}^{\prime}$ whenever $x$ and $y$ belong to (5.4) and (5.6) respectively. To see the following, recall the algebra structure on $B$ and $B^{\prime}$.

(1) (a) $\left(d a_{I^{\prime}}, 0\right) \cdot(c, c)=\left(d a_{I^{\prime}} \otimes c, 0\right)$.

(b) $(c, 0) \cdot(c, c)=\left(c^{2}, 0\right)=(0,0)$.

(c) $(0, c) \cdot(c, c)=(0,0)$.

(d) $\left(a_{I} \otimes b^{\prime},(-1)^{|I|} b^{\prime} a_{I}\right) \cdot(c, c)=(0,0)$.

(e) $\left(1 \otimes b^{\prime} a_{I}, b^{\prime} a_{I}\right) \cdot(c, c)=\left(1 \otimes b^{\prime} a_{I} c, b^{\prime} a_{I} c\right)=(0,0)$. 
(f) $\left(a_{I} \otimes b^{\prime}+\operatorname{sign}(\rho) a_{J} \otimes b^{\prime} a_{J^{\prime}}, 0\right) \cdot(c, c)=\left(a_{I} \otimes b^{\prime} c+\operatorname{sign}(\rho) a_{J} \otimes b^{\prime} a_{J^{\prime}} c, 0\right)=$ $(0,0)$.

(2) (a) $\left(d a_{I^{\prime}}, 0\right) \cdot\left(d a_{K} \otimes b^{\prime} a_{K^{c}}-c, 0\right)=\left(d a_{I^{\prime}} \otimes c, 0\right)$.

(b) $(c, 0) \cdot\left(d a_{K} \otimes b^{\prime} a_{K^{c}}-c, 0\right)=(0,0)$.

(c) $(0, c) \cdot\left(d a_{K} \otimes b^{\prime} a_{K^{c}}-c, 0\right)=(0,0)$.

(d) $\left(a_{I} \otimes b^{\prime},(-1)^{|I|} b^{\prime} a_{I}\right) \cdot\left(d a_{K} \otimes b^{\prime} a_{K^{c}}-c, 0\right)=\left(a_{I} d a_{K} \otimes b^{\prime 2} a_{K^{c}}-a_{I} \otimes b^{\prime} c, 0\right)=$ $(0,0)$.

(e) $\left(1 \otimes b^{\prime} a_{I}, b^{\prime} a_{I}\right) \cdot\left(d a_{K} \otimes b^{\prime} a_{K^{c}}-c, 0\right)=(0,0)$.

(f) $\left(a_{I} \otimes b^{\prime}+\operatorname{sign}(\rho) a_{J} \otimes b^{\prime} a_{J^{\prime}}, 0\right) \cdot\left(d a_{K} \otimes b^{\prime} a_{K^{c}}-c, 0\right)=(0,0)$.

(3) (a) $\left(d a_{I^{\prime}}, 0\right) \cdot\left(a_{\{1, \ldots, k\}} \otimes b^{\prime},(-1)^{k} b^{\prime} a_{\{1, \ldots, k\}}\right)=\left(d a_{I^{\prime}} a_{\{1, \ldots, k\}} \otimes b^{\prime}, 0\right)=(0,0)$.

(b) $(c, 0) \cdot\left(a_{\{1, \ldots, k\}} \otimes b^{\prime},(-1)^{k} b^{\prime} a_{\{1, \ldots, k\}}\right)=\left(a_{\{1, \ldots, k\}} \otimes c b^{\prime}, 0\right)=(0,0)$.

(c) $(0, c) \cdot\left(a_{\{1, \ldots, k\}} \otimes b^{\prime},(-1)^{k} b^{\prime} a_{\{1, \ldots, k\}}\right)=(0,0)$.

(d) $\left(a_{I} \otimes b^{\prime},(-1)^{|I|} b^{\prime} a_{I}\right) \cdot\left(a_{\{1, \ldots, k\}} \otimes b^{\prime},(-1)^{k} b^{\prime} a_{\{1, \ldots, k\}}\right)=(0,0)$.

(e) $\left(1 \otimes b^{\prime} a_{I}, b^{\prime} a_{I}\right) \cdot\left(a_{\{1, \ldots, k\}} \otimes b^{\prime},(-1)^{k} b^{\prime} a_{\{1, \ldots, k\}}\right)=(0,0)$.

(f) $\left(a_{I} \otimes b^{\prime}+\operatorname{sign}(\rho) a_{J} \otimes b^{\prime} a_{J^{\prime}}, 0\right) \cdot\left(a_{\{1, \ldots, k\}} \otimes b^{\prime},(-1)^{k} b^{\prime} a_{\{1, \ldots, k\}}\right)=(0,0)$.

(4) (a) $\left(d a_{I^{\prime}}, 0\right) \cdot\left(a_{K} \otimes b^{\prime} a_{K^{c}}+\operatorname{sign}(\rho) b^{\prime} a_{\{1, \ldots, k\}}, 0\right)$

$=\left(d a_{I^{\prime}} a_{K} \otimes b^{\prime} a_{K^{c}}+\operatorname{sign}(\rho) d a_{I^{\prime}} \otimes b^{\prime} a_{\{1, \ldots, k\}}, 0\right)$

$=\left(d a_{L} \otimes b^{\prime} a_{K^{c}}+\operatorname{sign}(\rho) d a_{I^{\prime}} \otimes b^{\prime} a_{\{1, \ldots, k\}}, 0\right) \in \mathcal{J}^{\prime}$.

(b) $(c, 0) \cdot\left(a_{K} \otimes b^{\prime} a_{K^{c}}+\operatorname{sign}(\rho) b^{\prime} a_{\{1, \ldots, k\}}, 0\right)=(0,0)$.

(c) $(0, c) \cdot\left(a_{K} \otimes b^{\prime} a_{K^{c}}+\operatorname{sign}(\rho) b^{\prime} a_{\{1, \ldots, k\}}, 0\right)=(0,0)$.

(d) $\left(a_{I} \otimes b^{\prime},(-1)^{|I|} b^{\prime} a_{I}\right) \cdot\left(a_{K} \otimes b^{\prime} a_{K^{c}}+\operatorname{sign}(\rho) b^{\prime} a_{\{1, \ldots, k\}}, 0\right)=(0,0)$.

(e) $\left(1 \otimes b^{\prime} a_{I}, b^{\prime} a_{I}\right) \cdot\left(a_{K} \otimes b^{\prime} a_{K^{c}}+\operatorname{sign}(\rho) b^{\prime} a_{\{1, \ldots, k\}}, 0\right)=(0,0)$.

(f) $\left(a_{I} \otimes b^{\prime}+\operatorname{sign}(\rho) a_{J} \otimes b^{\prime} a_{J^{\prime}}, 0\right) \cdot\left(a_{K} \otimes b^{\prime} a_{K^{c}}+\operatorname{sign}(\rho) b^{\prime} a_{\{1, \ldots, k\}}, 0\right)=(0,0)$.

(5) In this case $K \neq L^{c}$.

(a) $\left(d a_{I^{\prime}}, 0\right) \cdot\left(d a_{K} \otimes b^{\prime} a_{L}, 0\right)=(0,0)$, as $I^{\prime}, K \neq \emptyset$.

(b) $(c, 0) \cdot\left(d a_{K} \otimes b^{\prime} a_{L}, 0\right)=(0,0)$.

(c) $(0, c) \cdot\left(d a_{K} \otimes b^{\prime} a_{L}, 0\right)=(0,0)$.

(d) $\left(a_{I} \otimes b^{\prime},(-1)^{|I|} b^{\prime} a_{I}\right) \cdot\left(d a_{K} \otimes b^{\prime} a_{L}, 0\right)=(0,0)$.

(e) $\left(1 \otimes b^{\prime} a_{I}, b^{\prime} a_{I}\right) \cdot\left(d a_{K} \otimes b^{\prime} a_{L}, 0\right)=(0,0)$.

(f) $\left(a_{I} \otimes b^{\prime}+\operatorname{sign}(\rho) a_{J} \otimes b^{\prime} a_{J^{\prime}}, 0\right) \cdot\left(d a_{K} \otimes b^{\prime} a_{L}, 0\right)=(0,0)$.

(6) In this case $K \neq L^{c}$.

(a) $\left(d a_{I^{\prime}}, 0\right) \cdot\left(a_{K} \otimes b^{\prime} a_{L},(-1)^{|K|} b^{\prime} a_{K} a_{L}\right)=\left(d a_{K^{\prime}} \otimes b^{\prime} a_{L}, 0\right) \in \mathcal{J}^{\prime}$, where $K^{\prime}=I^{\prime} \cup K$.

(b) $(c, 0) \cdot\left(a_{K} \otimes b^{\prime} a_{L},(-1)^{|K|} b^{\prime} a_{K} a_{L}\right)=(0,0)$.

(c) $(0, c) \cdot\left(a_{K} \otimes b^{\prime} a_{L},(-1)^{|K|} b^{\prime} a_{K} a_{L}\right)=(0,0)$.

(d) $\left(a_{I} \otimes b^{\prime},(-1)^{|I|} b^{\prime} a_{I}\right) \cdot\left(a_{K} \otimes b^{\prime} a_{L},(-1)^{|K|} b^{\prime} a_{K} a_{L}\right)=(0,0)$.

(e) $\left(1 \otimes b^{\prime} a_{I}, b^{\prime} a_{I}\right) \cdot\left(a_{K} \otimes b^{\prime} a_{L},(-1)^{|K|} b^{\prime} a_{K} a_{L}\right)=(0,0)$. 
(f) $\left(a_{I} \otimes b^{\prime}+\operatorname{sign}(\rho) a_{J} \otimes b^{\prime} a_{J^{\prime}}, 0\right) \cdot\left(a_{K} \otimes b^{\prime} a_{L},(-1)^{|K|} b^{\prime} a_{K} a_{L}\right)=(0,0)$.

(7) (a) $\left(d a_{I^{\prime}}, 0\right) \cdot\left(d a_{J^{\prime}} \otimes c, 0\right)=(0,0)$.

(b) $(c, 0) \cdot\left(d a_{J^{\prime}} \otimes c, 0\right)=\left(d a_{J^{\prime}} \otimes c^{2}, 0\right)=(0,0)$.

(c) $(0, c) \cdot\left(d a_{J^{\prime}} \otimes c, 0\right)=(0,0)$.

(d) $\left(a_{I} \otimes b^{\prime},(-1)^{|I|} b^{\prime} a_{I}\right) \cdot\left(d a_{J^{\prime}} \otimes c, 0\right)=(0,0)$.

(e) $\left(1 \otimes b^{\prime} a_{I}, b^{\prime} a_{I}\right) \cdot\left(d a_{J^{\prime}} \otimes c, 0\right)(0,0)$.

(f) $\left(a_{I} \otimes b^{\prime}+\operatorname{sign}(\rho) a_{J} \otimes b^{\prime} a_{J^{\prime}}, 0\right) \cdot\left(d a_{J^{\prime}} \otimes c, 0\right)=(0,0)$.

(8) (a) $\left(d a_{I^{\prime}}, 0\right) \cdot\left(d a_{J^{\prime}} \otimes b^{\prime} a_{\{1, \ldots, k\}}, 0\right)=(0,0)$.

(b) $(c, 0) \cdot\left(d a_{J^{\prime}} \otimes b^{\prime} a_{\{1, \ldots, k\}}, 0\right)=(0,0)$.

(c) $(0, c) \cdot\left(d a_{J^{\prime}} \otimes b^{\prime} a_{\{1, \ldots, k\}}, 0\right)=(0,0)$.

(d) $\left(a_{I} \otimes b^{\prime},(-1)^{|I|} b^{\prime} a_{I}\right) \cdot\left(d a_{J^{\prime}} \otimes b^{\prime} a_{\{1, \ldots, k\}}, 0\right)=(0,0)$.

(e) $\left(1 \otimes b^{\prime} a_{I}, b^{\prime} a_{I}\right) \cdot\left(d a_{J^{\prime}} \otimes b^{\prime} a_{\{1, \ldots, k\}}, 0\right)=(0,0)$.

(f) $\left(a_{I} \otimes b^{\prime}+\operatorname{sign}(\rho) a_{J} \otimes b^{\prime} a_{J^{\prime}}, 0\right) \cdot\left(d a_{J^{\prime}} \otimes b^{\prime} a_{\{1, \ldots, k\}}, 0\right)=(0,0)$.

Proposition 5.9. The quotient map $\pi: \mathcal{D} \rightarrow \mathcal{D} / \mathcal{J}$ is a CDGA map and quasiisomorphism.

Proof. By Proposition 5.8, the elements of (5.6) form a graded basis of $\mathcal{J}$. Note that

(1) $d\left(b^{\prime} a_{\{1, \ldots, k\}}, b^{\prime} a_{\{1, \ldots, k\}}\right)=(c, c)$.

(2) $d\left(a_{I} \otimes b^{\prime} a_{I^{c}}-b^{\prime} a_{\{1, \ldots, k\}}, 0\right)=\left(d a_{I} \otimes b^{\prime} a_{I^{c}}, 0\right)$.

(3) $d\left(a_{I} \otimes b^{\prime} a_{J},(-1)^{|I|} b^{\prime} a_{I} a_{J}\right)=\left(d a_{I} \otimes b^{\prime} a_{J}, 0\right)$.

(4) $d\left(d a_{J^{\prime}} \otimes b^{\prime} a_{\{1, \ldots, k\}}, 0\right)=\left(d a_{J^{\prime}} \otimes c, 0\right)$.

Therefore we can conclude that $\mathcal{J}$ is an acyclic differential ideal.

Let $\bar{B}:=E^{\prime} \oplus_{\mathbb{Q}} B$. Then the natural map $E^{\prime} \oplus_{\mathbb{Q}} B \rightarrow E^{\prime} \otimes B$ is a differential graded module (DGM) chain map which induces an isomorphism in cohomology. Also $\bar{\phi}: \bar{B} \rightarrow C$ is a surjective DGM map so that

$$
a_{i} \rightarrow a_{i}, d a_{i} \rightarrow 0 \text { and } b^{\prime} a_{i_{1}} \cdots a_{i_{\ell}} \rightarrow b a_{i_{1}} \cdots a_{i_{\ell}}
$$

where $\left\{i_{1}, \ldots, i_{\ell}\right\} \subseteq\{1, \ldots, k\}$. Then the homotopy pull back of

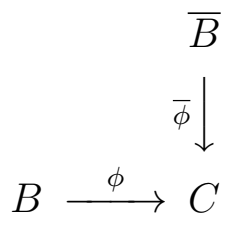

is $\overline{\mathcal{D}}=\operatorname{ker}(\bar{\phi}-\phi)$, where $\bar{\phi}-\phi: \bar{B} \oplus_{\mathbb{Q}} B \rightarrow C$. Note that $\overline{\mathcal{D}}$ is generated as graded module by the elements of the form

$$
\left\{\left(d a_{I}, 0\right),(c, 0),(0, c),\left(b^{\prime} a_{i_{1}} \cdots a_{i_{\ell}}, b^{\prime} a_{i_{1}} \cdots a_{i_{\ell}}\right)\right\}
$$

where $I,\left\{i_{1}, \ldots, i_{\ell}\right\} \subseteq\{1, \ldots, k\}$. Therefore, the natural inclusion $\iota_{D}: \overline{\mathcal{D}} \rightarrow \mathcal{D}$ is a differential graded module (DGM) map and quasi-isomorphism. 
Proposition 5.10. $\overline{\mathcal{D}}$ is a model for $S^{2 n} \#_{T^{k}} S^{2 n}$ and the set $(5.8)$ is a basis of $\overline{\mathcal{D}}$ over $\mathbb{Q}$.

Proof. The first statement follows from the well-known facts associated to homotopy pull-back of a diagram. For the rest, let $1 \leq i \leq 2 n-1$ and $\overline{\mathcal{D}}^{i}$ consists of $i$-th degree elements. Then dimension of $\overline{\mathcal{D}}^{i}$ and $H_{2 n-i}\left(S^{2 n} \#_{T^{k}} S^{2 n}\right)$ are same, and when $i=2 n$, $\overline{\mathcal{D}}^{i}$ is generated by $\{(c, 0),(0, c)\}$.

We remark that the map $\xi$ factors through $\iota_{D}$. Let $\eta: A \rightarrow \overline{\mathcal{D}}$ be the map such that $\xi=\iota_{D} \circ \eta$.

Proposition 5.11. The map $\eta: A \rightarrow \overline{\mathcal{D}}$ is injective $D G M$ and chain map.

Proof. Recall that differential on $A$ is zero and $\left\{\alpha_{I}: I \subseteq\{1, \ldots, k\}\right\}$ is a graded basis for $\widetilde{H}^{*} T^{k}$. Since $\alpha_{I}$ and $\alpha_{I}^{\#}$ are in complementary dimension, then

$$
\left\{\mu_{2 n}, s^{-1} \alpha_{I}, s^{-2 n+1} \alpha_{I}^{\#}: I \subseteq\{1, \ldots, k\}, I \neq \emptyset\right\}
$$

is a graded basis for $A$. Note that the map $\eta$ is same as $\xi$ as in (5.5). So $\eta$ is a graded module map and injectivity follows from the fact their image is a part of a basis as module over $\mathbb{Q}$.

It is a chain map since differential on $A$ is zero and differential on the image of each basis elements of $A$ is zero, that is $d\left(d a_{I}, 0\right)=(0,0), d(0, c)=(0,0)$, $d\left(b^{\prime} a_{i_{1}} \cdots a_{i_{\ell}}, b^{\prime} a_{i_{1}} \cdots a_{i_{\ell}}\right)=(0,0)$ for $\left\{i_{1}, \ldots, i_{\ell}\right\} \neq\{1, \ldots, k\}$.

We have the following commutative diagram of DGM.

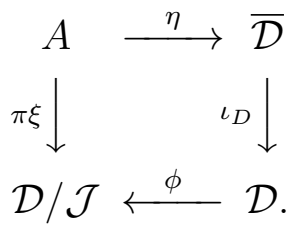

Proposition 5.12. The map $\pi \xi: A \rightarrow \mathcal{D} / J$ is a $C D G A$ map and quasi-isomorphism.

Proof. First we show that $\pi \xi$ is a quasi-isomorphism. So it is sufficient to show $\eta$ is quasi-isomorphism which is equivalent to show that $\overline{\mathcal{D}} / A$ is acyclic. The later is true since the ring $\overline{\mathcal{D}} / A$ can be generated by $\left\{(c, c),\left(b^{\prime} a_{\{1, \ldots, k\}}, b^{\prime} a_{\{1, \ldots, k\}}\right)\right\}$ as a graded module.

To show the other part, we need to show $\pi \xi$ is a chain map and $\pi \xi$ is an algebra map. Recall that $\left.\left\{s^{-1} a_{I}, s^{-2 n+1} a_{J}^{\#}, \mu_{2 n}: I, J \subseteq\{1, \ldots, k\}, I \neq \emptyset \neq J\right\}\right\}$ is a graded basis of $A$. We have the following:

(1) $d(\pi \xi)\left(s^{-1}\left(\alpha_{i_{1}} \cdots \alpha_{i_{\ell}}\right)\right)=d\left(d a_{i_{1}} a_{i_{2}} \cdots a_{i_{\ell}}\right)+\mathcal{J}=0+\mathcal{J}$.

(2) $d(\pi \xi)\left(s^{-2 n+1} \alpha_{I}^{\#}\right)=d\left(b^{\prime} a_{I^{c}}, b^{\prime} a_{I^{c}}\right)+\mathcal{J}=0+\mathcal{J}$, since $d\left(b^{\prime} a_{I^{c}}, b^{\prime} a_{I^{c}}\right)=(0,0)$ if $I \neq \emptyset$ and $d\left(b^{\prime} a_{I^{c}}, b^{\prime} a_{I^{c}}\right)=(c, c)$ if $I=\emptyset$.

(3) $d(\pi \xi)\left(\mu_{2 n}\right)=d(0, c)+\mathcal{J}=(0,0)+\mathcal{J}$.

Thus $\pi \xi$ is a chain map. Now to show $\pi \xi$ is an algebra map. We check this on the above graded basis of $A$. Recall the multiplication on $A$ from (1.2). Let $I, J \subseteq\{1, \ldots, k\}$ and $I=\left\{i_{1}, \ldots, i_{s}\right\}, J=\left\{j_{1}, \ldots, j_{t}\right\}$. 
(1) $\pi \xi\left(s^{-1} \alpha_{I} \cdot s^{-1} \alpha_{J}\right)=0=\pi \xi\left(s^{-2 n+1} \alpha_{I}^{\#} \cdot s^{-2 n+1} \alpha_{J}^{\#}\right)$. It follows from the multiplication in $A$.

$(2)$

$$
\begin{aligned}
\pi \xi\left(s^{-1} \alpha_{I}\right) \cdot \pi \xi\left(s^{-1} \alpha_{J}\right) & =\pi\left(d a_{i_{1}} a_{i_{2}} \cdots a_{i_{s}}, 0\right) \cdot \pi\left(d a_{j_{1}} a_{j_{2}} \cdots a_{j_{t}}, 0\right) \\
& =\pi\left(d a_{i_{1}} a_{i_{2}} \cdots a_{i_{s}} d a_{j_{1}} a_{j_{2}} \cdots a_{j_{t}}, 0\right) \\
& =0 \in \mathcal{D} / \mathcal{J}
\end{aligned}
$$

(3)

$$
\begin{aligned}
\pi \xi\left(s^{-1} \alpha_{I}\right) \cdot \pi \xi\left(s^{-2 n+1} \alpha_{J}^{\#}\right) & =\pi\left(d a_{i_{1}} a_{i_{2}} \cdots a_{i_{s}}, 0\right) \cdot \pi\left(b^{\prime} a_{J^{c}}, b^{\prime} a_{J^{c}}\right) \\
& =\pi\left(d a_{i_{1}} a_{i_{2}} \cdots a_{i_{s}} \otimes b^{\prime} a_{J^{c}}, 0\right) \\
& = \begin{cases}(c, 0)+\mathcal{J} & \text { if } I=J \\
(0,0)+\mathcal{J} & \text { otherwise in } \mathcal{D} / \mathcal{J} .\end{cases}
\end{aligned}
$$

(4)

Also, $\pi \xi\left(s^{-1} \alpha_{I} \cdot s^{-2 n+1} \alpha_{J}^{\#}\right)=\pi\left(\delta_{I J} \mu_{2 n}\right)=\delta_{I J}(0, c)=-\delta_{I J}(c, 0) \in \mathcal{D} / \mathcal{J}$.

$$
\begin{aligned}
\pi \xi\left(s^{-2 n+1} \alpha_{I}^{\#}\right) \cdot \pi \xi\left(s^{-2 n+1} \alpha_{J}^{\#}\right) & =\pi\left(b^{\prime} a_{I^{c}}, b^{\prime} a_{I^{c}}\right) \cdot \pi\left(b^{\prime} a_{J^{c}}, b^{\prime} a_{J^{c}}\right) \\
& =\left((-1)^{\left|I^{c}\right|} b^{\prime 2} a_{I^{c}} a_{J^{c}},(-1)^{\left|I^{c}\right|} b^{\prime 2} a_{I^{c}} a_{J^{c}}\right) \\
& =0+\mathcal{J} .
\end{aligned}
$$

(5) $\pi \xi(1 \cdot x)=\pi \xi(x)=\pi \xi(1) \cdot \pi \xi(x)$ for all basis element $x$ of $A$.

(6) (a)

$$
\begin{aligned}
\pi \xi\left(\mu_{2 n}\right) \cdot \pi \xi\left(s^{-1} \alpha_{I}\right) & =\pi(0, c) \cdot \pi\left(d a_{i_{1}} \cdots a_{i_{s}}, 0\right) \\
& =0+\mathcal{J} \\
& =\pi \xi\left(\mu_{2 n} \cdot s^{-1} \alpha_{I}\right)
\end{aligned}
$$

(b)

(c)

$$
\begin{aligned}
\pi \xi\left(\mu_{2 n}\right) \cdot \pi \xi\left(s^{-2 n+1} \alpha_{J}^{\#}\right) & =\pi(0, c) \cdot \pi\left(b^{\prime} a_{J^{c}}, b^{\prime} a_{J^{c}}\right) \\
& =\pi\left(0, c b^{\prime} a_{J^{c}}\right) \\
& =\pi(0,0) \\
& =\pi \xi\left(\mu_{2 n} \cdot s^{-1} \alpha_{I}\right)
\end{aligned}
$$

$$
\begin{aligned}
\pi \xi\left(\mu_{2 n}\right) \cdot \pi \xi\left(\mu_{2 n}\right) & =\pi(0, c) \cdot \pi(0, c) \\
& =(0,0)+\mathcal{J} \\
& =\pi \xi\left(\mu_{2 n} \cdot \mu_{2 n}\right) .
\end{aligned}
$$

In the rest of this section, we compute the cohomology ring of connected toric manifolds.

Theorem 5.13. Let $M$ and $N$ be $2 n$-dimensional toric manifolds. Then the cohomology ring of $M \#_{T^{k}} N$ is given by the ring $R\left(M, N, T^{k}\right)$ of (5.10). 
Proof. By Lemma $2.13 M \#_{T^{k}} N$ is homeomorphic to $M \# N \#\left(S^{2 n} \#_{T^{k}} S^{2 n}\right)$. Now applying the following process 3 times one can prove the theorem.

There are augmented map $\epsilon_{X}: H^{*}(X, \mathbb{Z}) \mapsto \mathbb{Z}$ and orientation classes $\mu_{X} \in$ $H^{2 n}(X)$, for $X \in\left\{M, N, M \# N,\left(S^{2 n} \#_{T^{k}} S^{2 n}\right)\right\}$. Let

$$
R=\left\{(a, b) \in H^{*}(M) \times H^{*}(N): \epsilon_{M}(a)=\epsilon_{N}(b)\right\}
$$

and $R(M, N)=R /\left\langle\left(\mu_{M},-\mu_{N}\right)\right\rangle$. Then the following cofibration

$$
S^{2 n-1} \rightarrow M \# N \rightarrow M \vee N
$$

implies that if $i+j=2 n$ with $i, j>0$ then the cup-product of $H^{i}(M)$ with $H^{j}(N)$ in $H^{2 n}(M \# N)$ is zero, but the product of $H^{i}(X)$ with $H^{j}(X)$ is the same as in the original manifold $X \in\{M, N\}$. Therefore, $H^{*}(M \# N, \mathbb{Z})$ is naturally isomorphic to $R(M, N)$.

Note that $M \# N$ is an oriented manifold. Let

$$
R^{\prime}=\left\{(c, d) \in H^{*}(M \# N) \times H^{*}\left(S^{2 n} \#_{T^{k}} S^{2 n}\right): \epsilon_{M \# N}(c)=\epsilon_{S^{2 n} \#_{T^{k}} S^{2 n}}(d)\right\}
$$

and

$$
R\left(M, N, T^{k}\right)=R^{\prime} /\left\langle\left(\mu_{M \# N},-\mu_{S^{2 n} \#_{T^{k}} S^{2 n}}\right)\right\rangle .
$$

By similar arguments as in the previous paragraph, $H^{*}\left(M \#_{T^{k}} N, \mathbb{Q}\right)$ is naturally isomorphic to $R\left(M, N, T^{k}\right)$.

Remark 5.14. The arguments in the proof of Theorem 5.13 is well-known. We represent it for the completeness. Explicit description of the cohomology ring of a toric manifold is given in [DJ91, BP02].

Now observing the algebra structure of $S^{2 n} \#_{T^{k}} S^{2 n}$, one may ask the following.

Question 5.15. Is the torus manifold $S^{2 n} \#_{T^{k}} S^{2 n}$ homeomorphic to the connected sum $\#_{i=1}^{t}\left(S^{m_{i}} \times S^{2 n-m_{i}}\right)$ of product spheres with the same cohomology algebra for some $t$ and $0<m_{i}<n+1$ ?

Acknowledgment: The authors would like to thank Pacific Institute of Mathematical Sciences and University of Regina.

\section{REFERENCES}

[AMPZ17] A. Ayzenberg, M. Masuda, S. Park, and H. Zeng. Cohomology of toric origami manifolds with acyclic proper faces. J. Symplectic Geom., 15(3):645-685, 2017.

[Ayz16a] A. Ayzenberg. Homology cycles in manifolds with locally standard torus actions. Homology Homotopy Appl., 18(1):1-23, 2016.

[Ayz16b] A. Ayzenberg. Locally standard torus actions and $h^{\prime}$-numbers of simplicial posets. $J$. Math. Soc. Japan, 68(4):1725-1745, 2016.

[BP02] V. M. Buchstaber and T. E. Panov. Torus actions and their applications in topology and combinatorics, volume 24 of University Lecture Series. American Mathematical Society, Providence, RI, 2002.

[BR01] V. M. Buchstaber and N. Ray. Tangential structures on toric manifolds, and connected sums of polytopes. Internat. Math. Res. Notices, (4):193-219, 2001.

[Dav83] M. W. Davis. Groups generated by reflections and aspherical manifolds not covered by Euclidean space. Ann. of Math. (2), 117(2):293-324, 1983. 
[DJ91] M. W. Davis and T. Januszkiewicz. Convex polytopes, Coxeter orbifolds and torus actions. Duke Math. J., 62(2):417-451, 1991.

[GK98] M. D. Grossberg and Y. Karshon. Equivariant index and the moment map for completely integrable torus actions. Adv. Math., 133(2):185-223, 1998.

[HM03] A. Hattori and M. Masuda. Theory of multi-fans. Osaka J. Math., 40(1):1-68, 2003.

[IFM13] H. Ishida, Y. Fukukawa, and M. Masuda. Topological toric manifolds. Mosc. Math. J., 13(1):57-98, 189-190, 2013.

[Joy12] D. Joyce. On manifolds with corners. In Advances in geometric analysis, volume 21 of Adv. Lect. Math. (ALM), pages 225-258. Int. Press, Somerville, MA, 2012.

[LS05] P. Lambrechts and D. Stanley. Algebraic models of Poincaré embeddings. Algebr. Geom. Topol., 5:135-182 (electronic), 2005.

[MP06] M. Masuda and T. Panov. On the cohomology of torus manifolds. Osaka J. Math., 43(3):711-746, 2006.

[PS15] M. Poddar and S. Sarkar. A class of torus manifolds with nonconvex orbit space. Proc. Amer. Math. Soc., 143(4):1797-1811, 2015.

[Yos11] T. Yoshida. Local torus actions modeled on the standard representation. Adv. Math., 227(5):1914-1955, 2011.

Department of Mathematics and Statistics, University of Regina, Regina, Canada

E-mail address: soumen@iitm.ac.in

Department of Mathematics and Statistics, University of Regina, Regina, Canada E-mail address: Donald.Stanley@uregina.ca 